\section{Gavetas e Introdução}

Prateleiras

\section{Dr. Guilherme Rodríguez da Silva}

Seção inaugurada em um de nossos primeiros números, em 1998, Gavetas e Prateleiras tem a finalidade de "resgatar textos importantes que tenham marcado época pelo seu significado no campo teórico, metodológico ou mesmo pela riqueza de um relato factual. Poderão ser inéditos (as gavetas) embora divulgados informalmente, o que é uma tradição em nossa área. .." ou, então, textos "precursores que tenham passado (quase) despercebidos ...(as prateleiras)". Nesta merecida homenagem a Guilherme Rodrigues da Silva publicamos neste número um texto de sua autoria publicado em 1984 pela Organização Pan Americana da Saúde (OPAS/ OMS). Transcrição de sua Conferência pronunciada no "Seminário sobre Usos y Perspectivas de la Epidemiologia", realizado em Buenos Aires (Argentina), em novembro do ano anterior. A publicação teve ampla divulgação na época de seu aparecimento mas ficou relegada em anos recentes. Conseguimos um exemplar graças à cortesia do Dr. José Roberto Ferreira, então responsável pelo Programa de Personal de Salud de la OPS e coordenador do Seminário. Foi editada, pela OPS/OMS, como Publicación No. PNPS 84-47 e podemos classificá-la inteira como prateleira em nossa tipologia de contribuições da RBE. Notável assinalar a criativa e inédita "Metodologia Del Seminário", introduzida pelo Dr. Ferreira, e que adotou um modelo matricial para promover as discussões "em dos vertientes em cuanto al contenido de las presentaciones y discusiones; uma que invólucro aspectos más generales (programas y servicios; formación; investigación); outra que incluyó lãs áreas de aplicación del conocimiento epidemiológico (evaluación; planificación; vigilância y control)".

Acreditamos que a contribuição do Prof. Guilherme, há mais de 20 anos, sobre "Formação de Pessoal em função dos usos e perspectivas da Epidemiologia" merece ser re-publicada e discutida à luz das recentes tendências da Epidemiologia brasileira. A publicação do artigo neste número da RBE será complementada, no próximo, por comentários que o contextualizem em particular comparando com o que dispõe o "IV Plano Diretor pa o desenvolvimento da Epidemiologia no Brasil”, publicado como Suplemento da RBE em dezembro de 2005, que pode ser acessado on-line em www.scielo.br/rbepid

O Editor

\title{
La formación de personal en función de los usos y perspectivas de la Epidemiología
}

En los últimos años se ha observado un renovado y profundo interés en discutir la epidemiología como instrumento para la producción de conocimientos y como técnica básica para la obtención de información esencial para la planificación y programación en materia de salud pública. Un buen ejemplo de ello es la reunión de la Asociación Internacional de Epidemiología, patrocinada conjuntamente por la OMS y la OPS y realizada en Las Croabas,
Puerto Rico, en septiembre de 1977 con la finalidad de examinar "las relaciones de las estrategias epidemiológicas" con los factores biológicos, sociales y ambientales y con la evaluación de la atención de salud y la regionalización de los servicios ${ }^{1}$.

La preocupación de estas organizaciones internacionales por la formación y el adiestramiento de personal en ese campo dió origen a varias propuestas que resultaron en la puesta en marcha de algunos 
programas importantes de capacitación y adiestramiento de personal ${ }^{2-7}$. En algunas instituciones latinoamericanas, particularmente en las facultades de salud pública ya se venía ofreciendo una programación más diversificada para capacitación en varios niveles, como en el caso de la Facultad de Salud Pública de Venezuela ${ }^{8}$.

¿Qué factores determinan la renovación del interés en los usos de la epidemiología, principalmente en lo que respecta a las aplicaciones, la planificación, la programación y la evaluación de los servicios de salud pública?

¿Por qué se concentra toda la atención en la epidemiología y se excluyen otros enfoques o técnicas de intervención y control, como las ciencias sociales aplicadas, las ciencias de gestión y administración sanitaria, etc.?

\section{CAMBIOS EN EL CAMPO DE LA SALUD PÚBLICA}

¿Guardaría esto alguna relación importante con las prácticas de salud pública y la organización social de los servicios de atención?

En los cinco primeros decenios del presente siglo, los programas de salud de los países latinoamericanos fueron organizados, por lo común, por técnicos de fuera, patrocinados por fundaciones extranjeras, y el trabajo se basó en programas verticales de control de las enfermedades endémicas que, por su naturaleza, ofrecían un mayor riesgo para la fuerza de trabajo y a menudo para los dirigentes provenientes de empresas de países colonizadores, que participaban en actividades agrícolas, de extracción mineral o vegetal, en la construcción de la infraestructura de transporte (por ejemplo, de las vías férreas al comienzo del siglo) y comunicaciones, necesarias para facilitar la actividad económica. Esos programas, que tenían por objeto enfermedades específicas como la malaria, la fiebre amarilla, la tuberculosis, la lepra, etc., tenían frecuentemente una clase de organización paramilitar, con personal uniformado, clasificado según un orden jerárquico y sometido a rigurosa disciplina ${ }^{9}$.

En un principio, la meta de estos programas era el control de las enfermedades endémicas. Después de terminar la Segunda Guerra Mundial, la ola de entusiasmo creada por la influencia de la OMS y la OPS sobre las posibilidades de éxito de dichos programas, llevó a los epidemiólogos a cargo de la planificación de los mismos, a proponer el establecimiento de un objetivo de control para la ambiciosa meta de erradicación de algunas enfermedades como la malaria, la viruela, la fiebre amarilla urbana y la frambesia. Pese a los buenos resultados de estos programas, al completo éxito logrado en el caso de la viruela y al éxito parcial, en el caso de la malaria, la fiebre amarilla urbana y la frambesia, el concepto de erradicación perdió gradualmente su credibilidad por varias razones enumeradas en la precitada revisión de Western ${ }^{9}$.

Junto con las modificaciones substanciales de la estructura social, a partir de los años treinta surgieron nuevas coyunturas sanitarias, que adquirieron mayor significado en los decenios de los años cuarenta y cincuenta y provocaron un cambio importante en la organización de los servicios de salud pública en varios países de América Latina. El crecimiento urbano, vinculado inicialmente con la expansión de la estructura de servicios relacionados con las economías exportadoras de productos primarios, y luego con el explosivo desarrollo industrial, representa el desplazamiento del campo hacia la ciudad, de un inmenso contingente de una fuerza de trabajo ociosamente ocupada en una agricultura de subsistencia, que dió origen a un patrón de integración típicamente capitalista periférico. Además de la masa obrera y de un ejército de reserva sometidos a continuo desplazamiento intraregional e interregional, se incrementó rápidamente la población marginal urbana que pasa a desempeñar también un papel fundamental en la producción de esas formaciones capitalistas tardías. El surgimiento de esos 
patrones de organización social en las ciudades, principalmente en los grandes centros metropolitanos formados en poco tiempo, creó la necesidad de establecer políticas sociales distintas de las predominantes hasta entonces, para agilizar el proceso de incremento del capital en esa fase de desarrollo urbano industrial.

Estas políticas dieron origen a programas de atención médica de previsión social y a nuevos patrones de servicios de salud pública. Estos se organizaron principalmente para controlar las endemias urbanas - la lepra, la tuberculosis y la elevada mortalidad infantil. Estos servicios, organizados inicialmente bajo la forma monovalente de dispensarios de tuberculosis y de lepra y centros de puericultura (higiene infantil), pasaron a integrar unidades locales de salud polivalentes, en años más recientes, en casi todos los países. La consolidación de esta nueva modalidad de organización de servicios no llega a ocurrir y comienza a observarse un verdadero proceso de desintegración y colapso de las redes de unidades locales de salud, en muchos sectores donde se establecieron. Son múltiples los factores determinantes de esa desintegración real o potencial: a) La falta de racionalidad de la organización dicotómica constituida por la salud pública frente a la atención médica, cuando ésta se ofrece principalmente por medio de servicios apropiados de previsión social como es el caso en México; b) la asignación preferencial de recursos financieros al sector de atención médica, especialmente en los países en que esos recursos se obtienen por medio de contribuciones relacionadas con la previsión social, pagadas total o parcialmente por empleados y empleadores, en detrimento del sector de salud pública costeado directamente por el Estado; c) la reducción de la atención prestada a los programas verticales de control de enfermedades por los gobiernos y las organizaciones internacionales, en parte porque el interés del capital financiero internacional canalizado a través de las empresas multinacionales desplazó su centro de interés de las zonas rurales para los centros urbanos; es decir, elaboración de productos primarios para fines de producción y consumo industrial; d) las presiones sociales que aumentan rápidamente, por medio de organizaciones sindicales y políticas de los trabajadores industriales del sector urbano, que se manifiestan en forma de demandas de servicios de atención médica instrumental (equipo, medicamentos) y no instrumental (conocimientos técnicos), canalizadas por medio de una campaña eficaz en los medios de información pública, que resulta en un proceso de comportamiento conocido con el nombre de "medicalización" de la sociedad, refuerzan el proceso de demanda, creciente e insatisfecha de servicios de atención médica, lo que da origen a un cambio radical en las políticas de salud de los distintos países.

Una posible solución para esos problemas parece ser la integración de los servicios básicos de salud pública de nivel local en los servicios de atención médica primaria y la oferta de los mismos por medio de las redes de unidades locales de salud que las administraciones de nivel estatal o provincial logran implantar. Estos servicios se llevarían a las zonas rurales remotas, por medio de unidades simplificadas, empleando principalmente personal auxiliar. El objetivo establecido en la Confererencia Internacional sobre Atención Primaria de Salud $^{10}$, realizada en Alma-Ata en 1978, en la que se recomendó que todos los pueblos del mundo alcancen en el año 2000 un nivel de salud que les permita llevar una vida social y económicamente productiva”, exige que los distintos países fijen una nueva estrategia nacional que les permita alcanzar las metas fijada en la propuesta de “Salud para Todos en el Año 2000”.

Se procura atribuir a los epidemiólogos y a la epidemiología la gran tarea de reorganización ${ }^{1,11}$, como instancia revigorizada de intervención social en el sector de salud. Esta debe ser la motivación en que se basa la nueva atención prestada a la epidemiología. Se pretende pasar de 
Graunt a Farr y de éste a Frost hasta llegar a la moderna y actual renovación de la confianza en la epidemiología, vista como epicentro de un sistema de información y de conformación de actitudes, que puede funcionar como un instrumento de control adecuado y eficaz.

Aunque se dude de la posible utilidad de la epidemiología como instrumento moderno de orientación doctrinaria en la organización de los nuevos servicios de atención de salud, no debe haber duda en lo que respecta a la función instrumental y técnica de la epidemiología que es una importante fuente de información para la organización de los servicios de salud. Como señaló acertadamente el Dr. Kerr White, "la finalidad de la información en el campo de la salud (o de la atención médica) es la identificación de problemas particulares y colectivos de salud, la evaluación de su importancia relativa, la estimación de la prevalencia y el costo social de los mismos, la identificación de la eficacia y los riesgos de las distintas formas de intervención y la evaluación del costo-beneficio de las medidas de prevención, control y restablecimiento de la salud. Más precisamente, la información sobre salud, que es distinta de la información médica, está relacionada con el deseo que tienen las poblaciones actuales y futuras de mejorar las condiciones de salud individuales y colectivas" ${ }^{12}$.

En 1981, la OPS reunió a un grupo de trabajo sobre Educación Continuada en Epidemiología, en Washington, D. C., para "evaluar las necesidades fundamentales identificadas en el plan de acción de Salud para Todos en el Año 2000, en el que la epidemiología desempeña una función esencial en la orientación de los servicios y la actuación del personal de salud" y para evaluar las consecuentes "necesidades de una mejor sistematización en la formación en epidemiología por medio de capacitación según los diversos niveles de servicios, en los próximos decenios"13.

Estas acciones parecen responder al llamado del Director General de la OMS, en su discurso de apertura de la reunión de 1978 sobre "Las estrategias epidemiológicas para la salud en un mundo cambiante"1: “... debemos formar una nueva generación de epidemiólogos orientados hacia Ia atención de salud en un contexto integral... “. Sin embargo, “. . .la utilización de conceptos, enfoques y métodos epidemiológicos no son pertinentes apenas para la formación del epidemiólogo profesional sino también para la capacitación de todo el personal de salud". En la presente reunión se debe volver a examinar esta propuesta de actuación teniendo en cuenta la tendencia observada en varios países a organizar una red de servicios básicos de salud en la que se integre la atención médica primaria, que es uno de los objetivos intermedios para alcanzar la meta de Salud para Todos en el Año 2000.

\section{NUEVOS USOS DE LA EPIDEMIOLOGÍA}

Una vez que los gobiernos de los países latinoamericanos adoptaron el objetivo común de alcanzar Salud para Todos en el Año $2000^{14}$ y que la OPS elaboró un amplio plan de acción para la instrumentación de esas estrategias ${ }^{15}$, surgieron como metas de orden más general a) el establecimiento de sectores y programas prioritarios; b) el desarrollo de la infraestructura de salud y c) la creación de sistemas de evaluación y auditoría.

Para cumplir con esos objetivos se consideran esenciales el conocimiento de la metodología epidemiológica y su efectiva utilización en los distintos niveles de organización de los servicios. Por ese motivo, se presta toda la atención posible a la formación y al adiestramiento en ese campo ${ }^{15}$.

¿Cuáles son realmente las tendencias de renovación de los usos de la epidemiología en la nueva estructura de servicios que todos esperamos ver surgir en los diversos países como consecuencia del plan de acción?

El virtual colapso de los programas verticales de control de enfermedades específicas, la especialización cada vez mayor 
en la esfera de las actividades de saneamiento y control del medio ambiente (resultante de la progresiva y acentuada división técnica del trabajo), que permite que las acciones eficaces de control ambiental (incluidas las de alimentos y de la contaminación ambiental) puedan continuar formando parte de los servicios locales e intermedios de salud, además de la predominancia de la atención médica respecto de los otros servicios de salud, en la asignación de prioridades y en la disputa por la asignación de recursos ${ }^{16}$, son factores que, en forma independiente o conjunta, han contribuido a la acentuada reducción de los servicios de salud pública en lo que respecta a la atención prestada a los grupos prioritarios, al control de las enfermedades transmisibles y a otras acciones locales. Por ende, no parece quedar alguna duda de que la única forma de revigorizar esos servicios será la integración de la atención médica primaria en los mismos, transformando la red básica de los servicios de salud pública, cuya eficacia se perdió progresivamente, en una red de servicios básicos de salud, en la que se pueda ofrecer de manera equilibrada, atención preventiva y curativa a todos los grupos sociales.

La función principal de la epidemiología en esta nueva estructura de servicios deberá consistir exactamente en garantizar el equilibrio entre las acciones de restablecimiento y prevención en la oferta de servicios, permitiendo y garantizando un enfoque demográfico en la organización de las acciones, a fin de evitar el predominio de la cura sobre la prevención de las enfermedades en el fomento de la salud.

Para que la epidemiología sea eficaz a nivel local es indispensable utilizarla también en los niveles regionales, intermedios y centrales como uno de los instrumentos $\left({ }^{*}\right)^{1}$ de:

Formulación de políticas sanitarias. Planificación de acciones a niveles nacional y internacional.
Programación de acciones a nivel nacional, regional y local.

Evaluación de los servicios y programas de:

a) salud pública, inclusive los programas de vigilancia epidemiológica;

b) atención médica primaria;

c) atención médica a niveles secundario y terciario.

1. Investigación científica, en la que se debe hacer hincapié en temas de importancia para la colectiva, y de aplicación más inmediata prácticas precitadas.

\section{PERSPECTIVAS}

El establecimiento de políticas de salud y atención médica tiene posibilidades demarcadas por los factores limitantes (restricciones) estructuralmente inherentes al modo capitalista de producción y organización social ${ }^{17}$; por ello, difícilmente nos dirigiremos a una organización de servicios integrados en su totalidad y racionalizada ya que el juego de las fuerzas predominantes en la mayoría de los países latinoamericanos, representadas por la vinculación de la atención médica priva$\underline{\mathrm{da}}$, los productores industriales de fármacos y equipo y los intereses económicos y políticos de los grupos sociales predominantes, con certeza mantendrá dentro de límites más o menos restrictos, las posibilidades de desarrollo de una estructura totalmente dirigida hacia intereses sociales más amplios.

Además de eso, las dificultades de la actual coyuntura de una crisis de dimensiones sin precedentes que azota fuertemente a un gran número de países, podría retardar mucho, si no obstaculizar, el deseado momento de transformación de los servicios de salud.

Es verdad que “... cualquiera que sea la superación de la crisis económica que vivimos, y parece que ya visualizamos los primeros síntomas de recuperación de las

${ }^{*}$ )Se debe tener en cuenta la parte subrayada porque creemos que el considerar la epidemiología como un instrumento exclusivo sería transformala en una panacea. 
economías centrales, es innegable que después de la crisis tendremos un escenario económico internacional totalmente diferente”. “...la inserción de las economías de América Latina y del Caribe en el contexto de la economía internacional sufrirá también una gran transformación"18.

Lamentablemente, no compartimos la visión optimista de White, para quien "la epidemiología nunca tuvo una mejor oportunidad de servir y proteger los intereses de la sociedad en el campo de la salud. La América Latina puede mostrar el camino"19. Pese a las dificultades previstas, sin embargo, la influyente actuación de la OPS ante los gobiernos de varios países latinoamericanos podrá suministrar el necesario apoyo político para las decisiones más centralizadas relativas a la codiciada organización de los servicios de salud. El apoyo técnico más necesario y útil será probablemente el exigido por la formación de recursos humanos, que se discutirá a continuación.

\section{FORMACIÓN DE RECURSOS HUMANOS}

No se trata apenas de la necesidad de preparar nuevas generaciones de epidemiólogos. Con certeza, eso se necesitará para inyectar nueva vida al sector que ha permanecido más o menos aislado, principalmente en la esfera de las facultades de salud pública y de los servicios verticales de control de las enfermedades transmisibles que quedan todavía.

Existe además una urgente necesidad de capacitación técnica en epidemiología, de personal de nivel universitario - planificadores, administradores, médicos especialistas en sanidad, enfermeras de salud pública, ingenieros sanitarios y sociólogos - así como también personal auxiliar para los servicios de nivel local de la red básica de salud.

Las contribuciones más específicas de los diversos relatores y ponentes de los temas especiales constituirán un material valioso para las discusiones de los grupos. La propuesta incluida a continuación se debe considerar como un programa de temas preliminar.

\section{PROYECTO DE PROGRAMA DE TEMAS PARA} DISCUSIÓN

I- LA PRÁCTICA EPIDEMIOLÓGICA ACTUAL FRENTE A LA PRÁCTICA QUE PODRÁ RESULTAR DEL PLAN DE ACCIÓN SALUD PARA TODOS EN EL AÑO 2000

1. Habida cuenta del conjunto de factores determinantes y limitantes en la esfera de:

a) las decisiones políticas nacionales;

b) las posibilidades y limitaciones en materia de apoyo internacional;

c) el estado actual de desarrollo de los servicios de salud;

d) la disponibilidad de recursos financieros

e) la disponibilidad de recursos humanos.

2. Existencia de nuevas dificultades en la organización de servicios, entre otros:

a) existencia de grandes masas urbanas marginalizadas en lo que respecta al consumo de bienes y servicios, que ejercen presión junto con los grupos más integrados a la actividad productiva, por razones de acceso a los servicios de salud y de exigencia de calidad de los mismos, principalmente de los de atención médica;

b) existencia de dudas en lo relativo a la mejor composición y las características de los servicios de atención médica primaria para los centros urbanos y sus zonas periféricas; por ejemplo, deben ser las características de esos servicios distintas de las de la atención primaria de salud propuesta para las zonas rurales remotas?;

c) dudas respecto de la posibilidad de debilitamiento de los programas preventivos con la incorporación de la atención primaria a los servicios básicos; 
d) dificultades de articulación de los servicios básicos y posibles dificultades en el establecimiento de relaciones funcionales con el sector de atención médica secundaria y terciaria controlado en la mayoría de las países por el sector privado de producción de servicios;

e) nuevos problemas de salud representados por los accidentes de trabajo y por la patología ocupacional

f) cambio a un primer plano de las enfermedades crónicas no infecciosas y de los problemas de salud relativos a la tercera edad, en la medida en que se consolidan los adelantos ya observados en materia de control de las enfermedades infecciosas transmisibles

3. Habida cuenta, además:

a) de las nuevas exigencias respecto de la capacitación de personal, especialmente de personal auxiliar y de agentes de salud con la incorporación de actividades de atención médica primaria más intensas y amplias que las acciones ya incluidas en los programas locales de salud pública tradicionales.

II. PERFIL PROFESIONAL DEL PERSONAL NECESARIO PARA EL PLAN DE SALUD PARA TODOS EN EL ANO 2000, TOMANDO COMO REFERENCIA EL PERSONAL ACTUALMENTE CONTRATADO, PARA FINES DE CONTRASTE Y COMPARACIÓN, Y PRESTANDO MAYOR ATENCIÓN AL CONTENIDO PEDAGÓGICO PARA FINES DEL ESTUDIO DE LA EPIDEMIOLOGÍA. CONSIDERANDO:

1. El nivel y la profesión del personal:

a) Médicos

b) Ingenieros sanitarios

c) Enfermeras

d) Asistentes sociales

e) Sociólogos, nutricionistas, educadores y otros profesionales

f) Personal auxiliar que ha tomado cursos de seis meses o más, por ejemplo, visitador sanitario, inspector sanitario, etc.

g) Personal auxiliar que ha recibido adiestramiento corto, por lo general, en servicio; por ejemplo, ayudantes, agentes de atención primaria de salud, etc.

2. La clase de programa educativo:

a) Postgrado a nivel de maestría o doctorado.

b) Especialización, inclusive residencia en medicina preventiva, medicina de la familia y otras.

c) Perfeccionamiento y educación continuada

d) Programas simplificados de tipo modular u otros.

III. EL CONTENIDO ESPECÍFICO DE LOS PROGRAMAS, EN EL CAMPO DE LA EPIDEMIOLOGÍA Y EN "CAMPOS COMPLEMENTARIOS”, TENIENDO EN CUENTA LA FORMACIÓN Y CAPACITACIÓN PARA:

A. Personal de nivel universitario:

1. Epidemiólogos. Investigaciones, enseñanza, servicio,

2. Administradores (inclusive planificadores)

3. Personal docente e investigaciones

4. Proveedores de servicios técnicos directos, médicos consultores, enfermeras de salud pública, nutricionistas, etc.

B. Personal auxiliar y agentes de salud.

1. Necesidad de adaptar los programas de estudio a las necesidades, la planificación, el proyecto respectivo o la prueba de programas y materiales didácticos.

2. Contenido modular con múltiples posibilidades de selección y exclusión de módulos específicos, según el país, la región, la clase de personal, etc.

3. ¿ Que material no epidemiológico se debe incluir? 
1. OPS. Las estrategias epidemiológicas para la salud en un mundo cambiante. Editorial, BoI Ofic. Sanit. Panam. 84(2):95-101, 1978.

2. OPS. Grupo de trabajo sobre la enseñanza de la epidemiología. Relato Final. Mimeografiado. Organización Panamericana de la Salud, Washington, D. C., noviembre de 1975.

3. OMS. Utilización de la epidemiología en la atención primaria de salud. Crónica de la OMS. 34: 18-21, 1980.

4. CAREC. Teaching of epidemiology. Surveillance Report, septiembre de 1981.

5. OMS. International training courses in epidemiology and control of communicable diseases. Wkly.Epidem. Rec. 52 245-2521, 1977.

6. OPS. Principios de epidemiología para el control de enfermedades. OPS, Washington, sin fecha.

7. Hernández, J. R. Cursos de Capacitación en epidemiología y control de enfermedades transmisibles. Mimeografiado. División de Epidemiología, Ministerio de Sanidad y Asistencia Social, Caracas, 1979.

8. Osuna, A.; Infante, R.; Borges R. y Padron, G.: La enseñanza de la epidemiología y de la estadística en los diferentes niveles de formación superior. Mimeografiado. Escuela de Salud Pública de Venezuela, Caracas, 1977.

9. Western, K. A.: Organization and administration of communicable disease control programs in developing countries. Bulletin of the Pan American Health Organization, 14: 65-76, 1980

10 Salud para Todos en el Año 2000. Declaración de Alma-Ata, Conferencia Internacional sobre Atención Primaria de Salud. Alma-Ata, URSS, 6-12 de septiembre de 1978.
11. Anderson, O. D.: Epidemiology in the planning process. British Columbia: Description of an experiment with a new model in epidemiology as a fundamental science: Oxford University Press, Nueva York, 1976

12. White, K. L. Information for health care: an epidemiologic perspective. Mimeografiado. Long-Range Working Groups on Biomedical and Health Information, Washington, D. C. 1980.

13. OPS, inédito. Carvalheiro, J. R. Información personal

14. OPS. Salud para todos en e1 ano 2000: Estrategias. Documento oficial 173. Oficina Sanitaria Panamericana, Washington, D. C. 1982.

15. OPS. Salud para todos en el ano 2000: Plan de acción para la instrumentación de las estrategias regionales. Documento oficial 179. Oficina Sanitaria Panamericana, Washington, D.C.

16. Terris, M.: The epidemiologic revolution, national health insurance and the role of health departments. Am. J. Pub. Health. 66: 1145-1164, 1976

17. Renald, M. Structural constraint to medicine in capitalistic societies. En Navarro, V. (ed.).

18. Guerra de Macedo, C.: Discurso en Panamá el 17 de agosto de 1983. Citado por Unidades de Epidemiología, OPS/OMS. Contribución de la epidemiología en el desarrollo de los programas de salud en América Latina. Seminario, OPS, Buenos Aires, 7-10 noviembre de 1983

19. White, K. L.: La epidemiología contemporánea: perspectivas y usos. Seminario sobre usos y perspectivas de la epidemiología, OPS, Buenos Aires, 7-10 de noviembre de 1983. 